\title{
SÍLABAS Y ACENTOS FUNDAMENTOS FONÉTICOS Y FONOLÓGICOS DEL RITMO
}

\author{
ESTEBAN TORRE
}

\begin{abstract}
T as sílabas y los acentos son, sin duda alguna, los elementos básicos del metro, y constituyen el armazón rítmico del verso (Torre 1999: 102). En el cómputo silábico y en el análisis de la distribución acentual residirían, en fin de cuentas, la clave y el objetivo último de los estudios métricos y rítmicos. La Métrica, o la Rítmica, sería, por consiguiente, la ciencia más sencilla del mundo. Y realmente lo es, al menos en lo que concierne a la vivencia personal y concreta de la experiencia literaria, tanto en el terreno de la creación poética como en el de la espontánea y atenta lectura.

Las cosas se complican cuando la obra poética deja de ser objeto de intuitivo deleite y se convierte en materia de disección, definición y taxonomía. El concepto de sílaba llega a hacerse sumamente problemático, en especial cuando se introduce el término de sílaba métrica para eludir, por ejemplo, la espinosa cuestión de las vocales en contacto. Navegando en el inseguro mar de las sinalefas y las dialefas, las diéresis y las sinéresis, la sílaba métrica se nos presenta a veces como un ente de razón, totalmente desvinculado de la realidad acústica. El número de sílabas -el número exacto de las sílabas de un verso- desciende claramente, en el sentir de algunos autores, al rango de lo meramente convencional.
\end{abstract}


Del mismo modo, en lo que respecta al acento, las cualidades físicas del sonido -tono, timbre, intensidad- no son tenidas suficientemente en cuenta, y se otorga en cambio una excesiva importancia a la duración silábica, identificándose las sílabas tónicas con las sílabas largas de la tradición grecolatina, y las átonas con las breves. En un reciente trabajo (Pardo 2001: 98), se ha llegado a afirmar que, en el endecasílabo común, la sílaba sexta acentuada, si pertenece a palabra oxítona, conserva «algo del alargamiento de sílaba acentuada final de verso o de hemistiquio», y que incluso el mismo recitado "parece confirmar la diferencia de duración».

Tengo el propósito de ocuparme, en sucesivos artículos, de estas y otras cuestiones relativas a la escansión del verso y el cómputo silábico, que ya fueron objeto de atención en anteriores trabajos (Torre 1999 y 2000). En este número inaugural de la revista Rhythmica, me limitaré a reiterarme en algunos aspectos fundamentales de la ciencia métrica, partiendo de ciertas aportaciones a la teoría del ritmo que provienen tanto de la Fonética Acústica, de base experimental, como de la Fonología Métrica, de filiación estructuralista y generativista. En el dominio de la Fonética Acústica, los conceptos que han tenido una mayor incidencia en relación con el cómputo silábico son los de isocronía e isosilabia.

Conviene precisar el valor de estos términos, porque, en diversos contextos, y según los distintos autores, pueden aparecer investidos de muy dispares significados. Por isocronía se entiende específicamente la isocronía acentual, esto es, la hipotética igualdad de las distancias temporales entre los acentos, independientemente del número de sílabas inacentuadas que existan entre ellos. La isosilabia designa, por el contrario, la igualdad en la duración de cada una de las sílabas. A este respecto, las lenguas naturales se han venido tradicionalmente clasificando de acuerdo con dos tendencias rítmicas opuestas: la tendencia a la isocronía acentual, o isocronía entre los pies acentuales, y la tendencia a la isosilabia, o isocronía silábica con anisocronía acentual. Dado el indiscutible dominio que la lengua inglesa 
ha venido ejerciendo en gran parte de la historia de los estudios sobre el ritmo (Cuenca Villarín 1997), no es extraño que las lenguas del primer grupo sean muchas veces denominadas stress-timed, y las del segundo grupo syllable-timed, siguiendo las pautas de Abercrombie (1967).

En la bibliografía especializada española, pueden encontrarse varias denominaciones para los términos ingleses stress-timed language y syllable-timed language. Así, Quilis y Fernández (1982) los traducen, respectivamente, como lenguas de ritmo acentualmente acompasado y lenguas de ritmo silábicamente acompasado. Toledo (1988) distingue entre lenguas de isocronía acentual y lenguas de anisocronía acentual. Cantin i Mas y Ríos Mestre (1991) las consideran como lenguas de compás acentual y lenguas de compás silábico. Para Almeida (1994), habría que hablar de lenguas de ritmo acentual, con isocronía localizada en el pie, como en las lenguas germánicas, y lenguas de ritmo silábico, con isocronía localizada en la sílaba, como en la mayoría de las lenguas románicas. Al primer grupo, caracterizado por la recurrencia acentual con distancias similares, se adscriben, en fin, lenguas tan dispares como el inglés, el ruso y el árabe. Al segundo grupo, definido por la similitud temporal entre las sílabas, se asignó el español, junto con otras lenguas romances, como el italiano, el francés y el catalán.

En estudios posteriores sobre el español, se rechazó esa tendencia a la isocronía silábica. Para Pointon (1978, 1980), no existe similitud temporal ni entre las sílabas ni entre las distancias temporales de los acentos o pies acentuales. Al no haberse podido comprobar isocronía silábica o acentual en la producción del habla, se defiende en su lugar (Allen 1973) una cierta isocronía en la percepción, como una tendencia del oyente a igualar perceptivamente las sílabas, o los pies acentuales, aunque éstos difieran considerablemente. Se ha llegado también a proponer la existencia de una isocronía profunda, es decir, una estructura rítmica subyacente, que sería modificada en el curso de la concreta realización del habla. Como prueba de esta supuesta isocronía subyacente, se aduce la existencia de dos hechos (Cantin i Mas y 
Ríos Mestre 1991): la introducción de acentos secundarios cuando la distancia entre las sílabas acentuadas excede de una cierta magnitud, y las omisiones de sílabas, que, más que como errores del habla, habrían de ser consideradas como perfeccionamiento de la estructura rítmica, ya que consiguen acompasar, igualando los periodos, el ritmo de la cadena fónica.

Las lenguas de ritmo acentual (stress-timed) se verían, además, afectadas por un fenómeno de compresión silábica, de forma que la duración de la sílaba tónica puede verse reducida en virtud de la influencia que sobre ella ejercen las sílabas átonas que la siguen y que la preceden. Lindblom (1975) observó que en la lengua sueca las sílabas inacentuadas, precedentes y siguientes, influyen decisivamente en la duración de la sílaba acentuada: a mayor número de sílabas inacentuadas, menor es la duración de la sílaba acentuada. El efecto de acortamiento de la sílaba tónica se hace más ostensible por la acción de la sílabas átonas siguientes; hablamos, en este caso, de "compresión anticipada”. Las lenguas de ritmo silábico (syllable-timed) mostrarían, en cambio, una escasa o nula compresión silábica.

En cualquier caso, la clasificación de las lenguas en dos grandes grupos, con características métricas bien definidas, dista mucho de constituir un cuerpo de doctrina sólidamente fundado. Y, desde luego, no puede basarse en esta división dicotómica de las lenguas una separación tajante entre la métrica silábica y la métrica acentual. Recientemente, Couper-Kuhlen (1993) ha insistido en que la isocronía no es meramente una cuestión de regularidad temporal, y sugiere un estrecho paralelismo entre los procesos de percepción visual y auditiva, lo cual implica el reconocimiento de grupos estructurados o gestalts en la captación de las imágenes acústicas.

El concepto de un ritmo isócrono, físico o subjetivo, viene desde luego determinado por un hecho incontrovertible: la existencia de un patrón rítmico, consistente en la alternancia de sílabas acentuadas e inacentuadas. El principio de alternancia, o lo que es lo mismo, de eufonía o eurritmia (prefiero esta grafía, eurritmia, a la académica euritmia, que puede llevar y lleva 
a una errónea pronunciación), parte de la observación de que las lenguas evitan la sucesión de sílabas con el mismo grado de tensión, haciendo que alternen las sílabas fuertes con las sílabas débiles.

En algunas lenguas, puede llegarse al desplazamiento acentual a fin de asegurar el principio de la eufonía. Así, en inglés, mediante la regla de “inversión yámbica”, se impide la sucesión de dos sílabas tónicas. Véase, por ejemplo, la pronunciación del conocido aeropuerto de Heathrow. El nombre de la localidad es Heathrów, con acentuación de la última sílaba; pero, en cambio, si nos referimos al aeropuerto, la pronunciación ha de ser Héathrow Áirport, con acento en la primera sílaba. En la lengua española, Eduardo Benot había llamado ya la atención sobre el hecho de que, en el verso endecasílabo, "no deben concurrir inmediatas i tocándose dos sílabas intensas y vigorosas”. Y, a renglón seguido, especifica:

Esto es verdad cuando el primer acento es obstruccionista del segundo, i no deja sentir el ritmo métrico; pero un hábil versificador, cuando no tenga a mano más que sílabas naturalmente acentuadas, logrará que el acento natural constituyente ofusque i desvanezca (con la resultante de intensidad natural i de posición que sabrá darle para hacer sentir el ritmo) al acento contiguo supernumerario, cuya intensidad no podrá ya perjudicar a la constitución endecasilábica (Benot 1892: I, 198).

Es evidente que el acento no constituye en el verso español una entidad absoluta, sino relativa (Torre 1986: 39). La sílaba acentuada lo es siempre en relación a un entorno. Una sílaba, con relieve acentual en la palabra aislada, puede perderlo en la línea del verso. Y, a la inversa, una sílaba menos relevante puede experimentar un incremento acentual por su posición en el verso, la entonación o el énfasis que se ponga en la lectura para destacar el sentido. En el verso inglés, Susanne Woods advierte que, dadas dos sílabas consecutivas, una deberá ser relativamente más acentuada que la otra («one will be relatively more 
accented than the other») (Baker 1996: 286). Y lo mismo ocurre en el verso italiano, donde se hace notar la imposibilidad de que existan dos acentos rítmicos consecutivos (Pazzaglia 1990: 82), ya que, si se encuentran dos acentos, uno de los dos debe ser suprimido o trasladado. Con todo, algún autor (Bausi y Martelli 1993: 27) defiende la tesis contraria: podría haber dos sílabas adyacentes provistas ambas de acento rítmico, esto es, los versos $-\mathrm{y}$, en concreto, los versos italianos- podrían presentar dos ictus consecutivos («i versi italiani possono presentare due ictus consecutivi»).

En realidad, se trata de una cuestión puramente conceptual y terminológica. El mayor relieve de la sílaba acentuada se refiere siempre a un entorno, a un antes y a un después menos acentuados. Entra así en juego el concepto de alternancia, que ha sido desarrollado por investigadores de signo muy diverso, tanto en el aspecto teórico (Fonología Métrica) como en el práctico y experimental (Fonética Acústica). En Fonología Métrica, el acento se entiende como una relación sintagmática entre dos componentes, uno fuerte y otro débil, que, como ya hemos indicado, no constituyen entidades absolutas, sino relativas. En torno al contraste relativo de tensión -o prominencia- entre estos dos componentes, se organizan las reglas métricas.

El deseo de ofrecer una precisa gradación acentual llevó a algunos autores al establecimiento de distintos niveles en el acento. Así, en 1900, Otto Jespersen ("Notes on Metre”, reimpreso en Gross 1979), aun reconociendo que en realidad existe una gama infinita en el acento (stress), distinguió cuatro niveles en la lengua inglesa, marcados numéricamente: 4, fuerte (strong); 3, semifuerte (half-strong); 2, semidébil (half-weak); y 1, débil (weak). Otros autores (Trager y Smith 1951) establecieron un sistema parecido de cuatro niveles acentuales: primario (primary), secundario (secondary), terciario (tertiary) y débil (weak). Este sistema, empleado por metristas como Seymour Chatman (1965), pretendería realizar una más fina discriminación del ritmo, al menos desde un punto de vista teórico. 
En la práctica, sin embargo, se ha puesto de manifiesto (Baker 1996: 31) el carácter inviable de esta gradación acentual. Si ya el sistema convencional de dos niveles acentuales (débil y fuerte) puede resultar en ocasiones discutible, un sistema de cuatro niveles multiplicaría los desacuerdos y sólo nos llevaría a crear una confusión todavía más elaborada. Una fórmula de compromiso es la que distingue tres grados en el acento (Hayes 1995: 16): acento principal (main stress), acento secundario (secondary stress) y sílaba inacentuada (stressless).

En los planteamientos teóricos de la Fonología Métrica (Kager 1989, Vish 1990, Hayes 1995, Toledo 1996), se propuso que las representaciones mentales de los patrones de acento estarían organizadas jerárquicamente y no en forma lineal. Una cuadrícula métrica abstracta (metrical grid) representaría las relaciones acentuales en la palabra o en la frase. El nivel acentual, en una sílaba dada, viene simbolizado por la altura de una columna de marcas. La relativa prominencia $(\mathrm{s}=$ strong, fuerte; $\mathrm{w}=$ weak, débil) de cada uno de los segmentos aparece representada en forma de árbol:
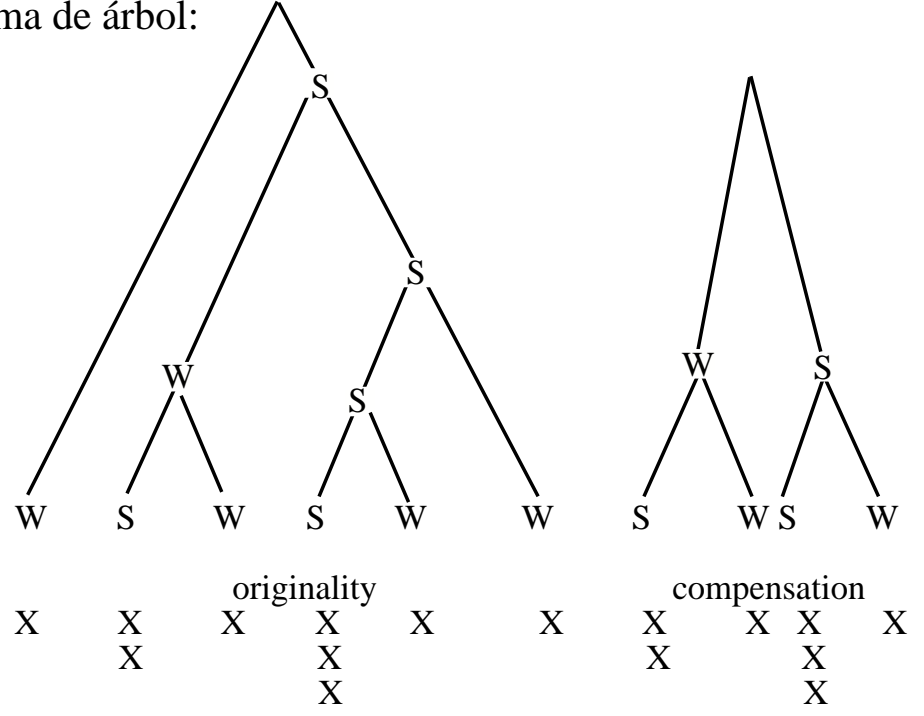
Cada sílaba recibe una marca en el nivel más bajo. Las sílabas que sólo llevan una marca se perciben como inacentuadas, y las que tienen dos o más marcas se perciben como acentuadas, bien con acento principal, bien con acentos secundarios. Puede tener lugar un desplazamiento acentual (stress shift), debido a la proximidad de un elemento más prominente, como ya se indicó en el caso de la inversión yámbica: Heathrów y Héathrow Áirport. Al cambiar el lugar del acento, y por tanto, la configuración de la cuadrícula, cambia también la distribución de los segmentos "s" y "w" en el árbol. Es lo que ocurre, por ejemplo, cuando la palabra thirteen pasa a formar parte de la frase thirteen men. En la palabra aislada, la primera sílaba es menos prominente que la segunda: thirtéen. En la frase, la mayor prominencia de men produce la inversión del acento en la palabra contigua, cuya primera sílaba es ahora la más prominente: thírteen. Se puede esto ilustrar de la siguiente manera:

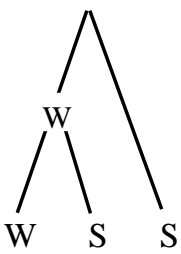

thirtéen mén $\begin{array}{lll}X & X & X \\ & X & X \\ & & X\end{array}$

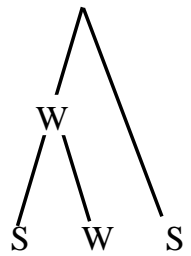

thírteen mén $\begin{array}{lll}X & X & X \\ X & & X \\ & & X\end{array}$

En español, la alternancia ha sido documentada por autores como Navarro Tomás (1966), Gili Gaya (1975), Harris (1983), Halle y Vergnaud (1987) y Toledo (1996). Se han descrito dos patrones de acentuación en el interior de la palabra:

a) gèneratívo, gràmaticalidád.

b) genèratívo, gramàticalidád. 
Los acentos secundarios pueden aparecer: (a) en la sílaba inicial, o (b) en las sílabas impares, contando a partir del acento principal hacia la izquierda. Según Harris, el patrón (a) sería coloquial, mientras que el (b) tendría un tinte retórico. Para Gili Gaya, estas normas serían más bien la manifestación de tendencias lingüísticas particulares (primer tipo) y universales (segundo tipo). Cuando no hay una causa en contra, como, por ejemplo, una focalización precisa, esto es, una carga enfática extraordinaria sobre algún segmento (Canellada y Madsen 1987), lo más general es que el discurso se desarrolle con alternancia de acentos.

No hay duda de que, por poco empeño que se ponga en ello, colocando acá y allá, cada dos o tres sílabas, acentos primarios y secundarios, se consigue una perfecta segmentación del discurso en troqueos y dáctilos. Y, si fácil es segmentar un texto en prosa, estableciendo en él una división en grupos fónicos o pies acentuales, mucho más sencillo ha de resultar el análisis rítmico del verso, “más medido y más regulado”, según autores como María Josefa Canellada y John Kuhlmann Madsen (1987: 154-156), para quienes «el verso es la cristalización de la prosa, más amorfa».

Dividen los versos estos autores en varios grupos. Uno de ellos se caracteriza por poseer un ritmo que «se mantiene a base solamente de tiempos musicales, fuertes y débiles», sin que exista «igualdad de sílabas en el verso, ni de cláusulas». Y en este grupo incluyen la siguiente composición de Juan Ramón Jiménez:

Siento que el barco mío ha tropezado, allá en el fondo, con algo grande.

$$
\begin{aligned}
& \text { ¡Y nada } \\
& \text { sucede! Nada... Quietud... Olas... } \\
& \text { ¿Nada sucede; o es que ha sucedido todo, } \\
& \text { y estamos ya, tranquilos, en lo nuevo? }
\end{aligned}
$$

Sorprende que se cataloguen como irregulares (sin igualdad de sílabas ni de cláusulas, esto es, fuera de toda norma en lo 
relativo al cómputo silábico) unos versos que se encuentran perfectamente dentro de los cauces del más exacto ritmo endecasilábico. Se trata, en efecto, de una serie de seis versos, de los que el tercero aparece escalonado: «con algo grande. ¡Y nada». Obviamente, ni la puntuación ni el escalonamiento impiden la sinalefa: «con-al-go-gran-de $i$-na-da». Constituye, así pues, un heptasílabo, al igual que el verso primero. El segundo y el cuarto son eneasílabos; el quinto, un alejandrino, compuesto por dos hemistiquios heptasilábicos («¿Na-da-su-ce-de-oés-[0] / quehasu-ce-di-do-to-do»); y el sexto y último, un verso endecasílabo. Pero Canellada y Madsen ignoran la existencia del escalonamiento del verso tercero, y consideran el quinto como un verso compuesto por dos hemistiquios de cinco y ocho sílabas. Por otra parte, el uso distributivo que hacen de los acentos, principales (') y secundarios ('), es totalmente caprichoso.

Es evidente que los meros criterios fonéticos y fonológicos no nos proporcionan un instrumento de análisis que pueda ser aplicable, sin más, al estudio de la métrica. Los mismos conceptos de sílaba y acento son problemáticos. La noción de sílaba es tan poco clara, desde un punto de vista científico, como intuitiva en la conciencia de todo hablante (De Rosa y Sangirardi 1996: 20). Si para la Real Academia Española (1973: 12) se trata efectivamente de una noción más intuitiva que científica, para la Academia Húngara la sílaba carece prácticamente de función lingüística y consiste tan sólo en una unidad rítmica, de base fisiológica y acústica, cuya existencia depende de la convención y el uso de una determinada comunidad lingüística (Nyéki 1973: 138).

Para el metricista italiano Pietro Beltrami (1996: 31), que considera asimismo la sílaba como unidad rítmica -sería preferible llamarla elemento rítmico, reservando para el verso la denominación de unidad rítmica-, es desde luego una noción difícil de definir científicamente. Aunque, a pesar de la oscuridad de la noción de sílaba, no hay duda de que en el habla alienta una especie de pulso o de latido (pulse phenomenon) que se percibe antes y mejor que los fonemas mismos (Lotz 1974: 972). Di Girolamo, inspirándose en la métrica generativa de Halle y Keyser (1971), 
ha propuesto sustituir el término sillaba metrica por el de posizione: «La posizione è l'unità minima del verso» (Di Girolamo 1976: 22). Un número fijo de posizioni conformaría el modelo métrico de cualquier tipo de verso.

A la imprecisión del concepto de sílaba viene a añadirse el problema de los diptongos, y, en el caso particular del verso francés, el de la $e$ muda. Ambos constituyen «les points vulnérables du syllabisme» (Kibédi Varga 1977: 76). El carácter inestable de la $e$ muda francesa amenaza, en efecto, el silabismo en sus mismos fundamentos (Kibédi Varga 1977: 80). Algo similar ocurre en el estado actual de la lengua portuguesa, donde es habitual la elisión de las vocales átonas. En alemán, las frecuentes contracciones que se producen en el lenguaje ordinario plantean un análogo problema.

Ninguna de estas circunstancias se dan, desde luego, en el verso español; pero sí está presente la cuestión del encuentro de vocales (Torre 1986: 27-32), bien entre palabras (sinalefa y dialefa), bien en el cuerpo de una misma palabra (diéresis y sinéresis). Si los términos sinalefa y dialefa son utilizados de una manera unívoca por la generalidad de los tratadistas, no ocurre lo mismo en el caso de la diéresis y la sinéresis. Algunos autores dan una definición correlativa (Spongano 1974: 18, Pazzaglia 1990: 42) de ambos términos: si dos vocales contiguas, en el interior de una palabra, forman una sola sílaba métrica, existiría sinéresis; el fenómeno contrario sería la diéresis. Se iguala, así, la diéresis al hiato; y la sinéresis, al diptongo. La preceptiva española tradicional consideraba estos fenómenos como licencias poéticas, es decir, como transgresiones de las leyes gramaticales, que se permitían sólo en nombre de las necesidades métricas. Pero pienso que no es así. Se trata simplemente de un hecho de habla, que el poeta utiliza de una manera intuitiva y espontánea.

También en los tratados de métrica italiana, se conceptúan frecuentemente estos fenómenos como transgresiones de la norma. Así, diéresis y sinéresis indicarían una escansión distinta de la normal (Beltrami 1996: 34), y consistirían en pronunciar dos vocales contiguas en el interior de la palabra de forma diferente 
a la de la norma italiana (Orlando 1993: 26). Se daría el fenómeno de la diéresis cuando se hacen artificialmente dos sílabas de lo que, en la lengua, constituye una sola (Menichetti 1986: 17). En alguna ocasión, la interpretación que recibe este fenómeno es, en verdad, sorprendente. Es lo que ocurre, por ejemplo, con el siguiente endecasílabo del Dante (Par. XXXI, 37):

$$
\text { io, che al divino dall'umano... }
$$

La lectura correcta es, sin duda alguna, la que introduce una diéresis en io y una dialefa entre che y al. Pues bien, Mario Fubini (1975: 37) afirma que lo que existe no es una diéresis, sino una "sílaba silenciosa" («non una dieresi ma una sillaba silenziosa»). Cuando el número de sílabas de un endecasílabo no es el de once, entonces «la voce tende o a eliminare o a passare in sordina le sillabe eccessive, o a porre delle pause dove le sillabe sono mancanti» (Fubini 1975: 39). La presencia de esta pretendida sílaba silenciosa, interpretada como una pausa que sustituye a una sílaba, fue oportunamente tildada de extravagante por parte de la misma crítica italiana (Menichetti 1986: 7).

A decir verdad, la escansión del verso puede entrañar algunas dificultades, aun en circunstancias aparentemente sencillas. Consideremos, por ejemplo, estos versos de Juan Ramón Jiméez:

Ya están las rosas primeras dispuestas a embriagarnos.

La lectura del primer verso como octosílabo no ofrece la menor dificultad. Nadie dudaría en realizar la sinalefa entre "ya” y “es-” (de “están”): “yaes-tan-las-ro-sas-pri-me-ras”. En cambio, en el segundo verso, que también es un octosílabo que forma parte de la serie arromanzada, pueden surgir discrepancias a la hora de elegir entre dos posibles interpretaciones:

a) Diéresis en "embrïagarnos” y sinalefa entre "a” y “em-" (de “embrïagarnos”): “dis-pues-ta-saen-bri-a-gar-nos”. 
b) Dialefa entre “a” y “em-” (de “embriagarnos”, sin diéresis): "dis-pues-ta-sa-em-bria-gar-nos”.

La primera lectura es, a mi modo de ver, la correcta; pero no dispongo de un procedimiento objetivo que me permita demostrarlo científicamente.

Con el fin de obtener un correlato físico, objetivo, de los fenómenos fonéticos implicados en la escansión del verso, he llevado a cabo diversas experiencias en el Laboratorio de Fonética de la Facultad de Filología, en la Universidad de Sevilla. Como más representativas, ofrezco aquí las gráficas que recogen respectivamente el oscilograma y la curva melódica, realizadas por ordenador con el sistema xwaves+ de Entropic para entorno UNIX, a partir de los siguientes versos de Federico García Lorca:
¿Qué es aquello que reluce por los altos corredores?
Cierra la puerta, hijo mío; acaban de dar las once.

El oscilograma, representado en la primera gráfica de las Figuras I-IV, recoge las intensidades de los sonidos emitidos en el decurso de la lectura de cada uno de los versos. Como es sabido, la intensidad acústica es la cantidad de energía que, en la unidad de tiempo, atraviesa una unidad de superficie situada perpendicularmente a la dirección de propagación de las ondas sonoras. Se mide en Watios $/ \mathrm{m}^{2}$ y es proporcional a la amplitud de la onda. Se observa, en las gráficas, en el eje vertical de las ordenadas. La duración se mide, en fracciones de segundo, sobre el eje horizontal de las abscisas.

El espectrograma de la curva melódica, representado en la segunda gráfica de las FiguRAS I-IV, nos da el tono musical, esto es, la frecuencia o número de ciclos por segundo de los sonidos emitidos. Se mide en Hercios sobre el eje de ordenadas. Es de advertir que la frecuencia que se recoge en esta curva melódica es la frecuencia fundamental, que es precisamente la que cons- 
tituye el tono musical, si bien existe un cierto número de armónicos, que determinan el timbre y que no se tienen en cuenta en el presente estudio. En la Figura v, puede apreciarse la gráfica del tono musical en sentido estricto, ya que se recogen concretamente varias notas (cuarta ascendente y cuarta descendente) de la escala musical.

En el primer verso (FIGURA I), que es un octosílabo de ritmo marcadamente trocaico, podemos observar en el oscilograma cómo las sílabas impares presentan una intensidad ligeramente superior a la de las sílabas pares. Especialmente, entre la sílaba tercera y la cuarta existe una marcada diferencia de intensidad acústica, en estrecha relación con el énfasis que se pone en la lectura del deíctico “aquello”. Por su parte, la línea melódica que se observa en el espectrograma refleja muy bien la alternancia tonal, con una importante caída de la frecuencia fundamental de la última sílaba postónica con respecto a la tónica precedente.

El acento rítmico está en función, por lo tanto, no sólo de la intensidad acústica, sino también de la frecuencia fundamental o tono de cada una de las sílabas. La cuantificación temporal, en fracciones de segundo, carece de relevancia.

El oscilograma del segundo verso (FIGURA II) es el que más claramente refleja la mayor intensidad de las sílabas impares, siendo ostensible la materialización acústica del ritmo trocaico. Aquí también la sílaba tercera y la cuarta acusan la máxima diferencia de intensidad acentual, en íntima conexión con el contenido semántico del la palabra "altos”. La tercera sílaba presenta, asimismo, la mayor altura tonal de la serie silábica del verso.

Tanto del oscilograma como del espectrograma del verso tercero (FIGURA III) podemos extraer los datos más significativos. También en este caso, en el ritmo del verso, que es ahora dactílico, es el segundo pie el más marcado. Obsérvese que existe sinalefa entre "-ta” (de "puerta") e "hi-" (de "hijo"). En virtud de la sinalefa, "puerta, hijo" (puér-tahi-jo) es una secuencia trisilábica con acento sobre la primera sílaba, tal como se recoge en la gráfica del oscilograma. El acento de la palabra aislada "hijo" queda anulado en la línea del verso. 
Pero, además de la intensidad acústica, hay que tener en cuenta las diferencias tonales. La palabra "hijo" conserva una cierta independencia acentual con respecto a la palabra "puerta” en virtud de la frecuencia acústica, no de la intensidad. El espectrograma de frecuencias recoge lo que ya habíamos observado por audición directa: un cambio de tono entre "puer-" y "-tahi-", lo cual confiere una matización acústica a la segunda sílaba métrica de la secuencia trisilábica, supliendo así la pérdida de intensidad acentual.

El análisis acústico de las sílabas finales, “mí-o”, presenta un gran interés. El oscilograma no acusa prácticamente diferencia alguna entre las intensidades de las dos sílabas que constituyen la palabra "mío”. Por otro lado, al no existir entre ambas sílabas ningún tipo de cerrazón consonántica, no se interrumpe en ningún momento el fluir sonoro, como se aprecia en la gráfica. Ahora bien, si el oscilograma no contiene señal alguna que nos permita establecer unos límites silábicos, el espectrograma muestra con toda claridad la gran diferencia tonal que existe entre ambas sílabas. En otras palabras: el correlato físico del acento es, en este caso, el tono.

Un desdoble del tono, como el que acabamos de señalar, no implica necesariamente una duplicidad silábica. En la línea melódica correspondiente al cuarto y último verso (FIGURA IV), puede apreciarse cómo la sílaba final tónica, “on-” (de “once”), sufre un desdoblamiento tonal muy marcado. El segundo tono corresponde, según se aprecia en la gráfica del espectrograma, a la sonoridad de la coda nasal; pero la unidad de la sílaba permanece inalterada.

Es muy ilustrativa, por otra parte, en el oscilograma de este mismo verso, la distribución de las intensidades acústicas en tres pies métricos bien definidos: acaban / de dar / las once. Utilizando, mutatis mutandis, la terminología de la métrica clásica, podríamos decir que el verso consta de los siguientes pies: anfíbraco, yambo y anfíbraco. Cualquier otro tipo de segmentación, que considerando, por ejemplo, como anacrusis la sílaba átona inicial de “acaban”, tratara de adscribir el verso a un ritmo mixto 
dactílico-trocaico, supondría no sólo el olvido del componente sintáctico-semántico del verso, sino también un desconocimiento del mismo sustrato fónico que la gráfica del oscilograma pone claramente de manifiesto.

De pies métricos y grupos fónicos me he ocupado en otro lugar (Torre 1999: 15-26). Sólo quiero indicar aquí que la confusión de estos segmentos con los hemistiquios ha dado también origen a inexactas apreciaciones. Así, por ejemplo, para Maurice Grammont (1974: 49-60) los “cortes” (coupes) que tienen lugar entre los grupos rítmicos, esto es, entre los pies métricos, se confunden con la pausa que marca la "cesura" (césure). Para este autor, la cesura no sería más que un simple corte (une simple coupe). Estoy convencido de que la falta del adecuado reconocimiento de la pausa -o la cesura- como factor demarcativo, junto con las ya señaladas imprecisiones en la segmentación silábica del verso, es causa de frecuentes errores en el cómputo silábico y punto de arranque de un larvado relativismo en los estudios métricos, según el cual el silabismo se reduciría a un sistema meramente aproximativo, del que no tendríamos en absoluto un conocimiento intuitivo y directo, y mucho menos científico. Un análisis fonético y fonológico de la línea del verso, profundo y detenido, sin apriorismos ni lastres conceptuales, contribuirá poderosamente a su mejor conocimiento, y al disfrute, en última instancia, de eso que hemos convenido en llamar poesía. 

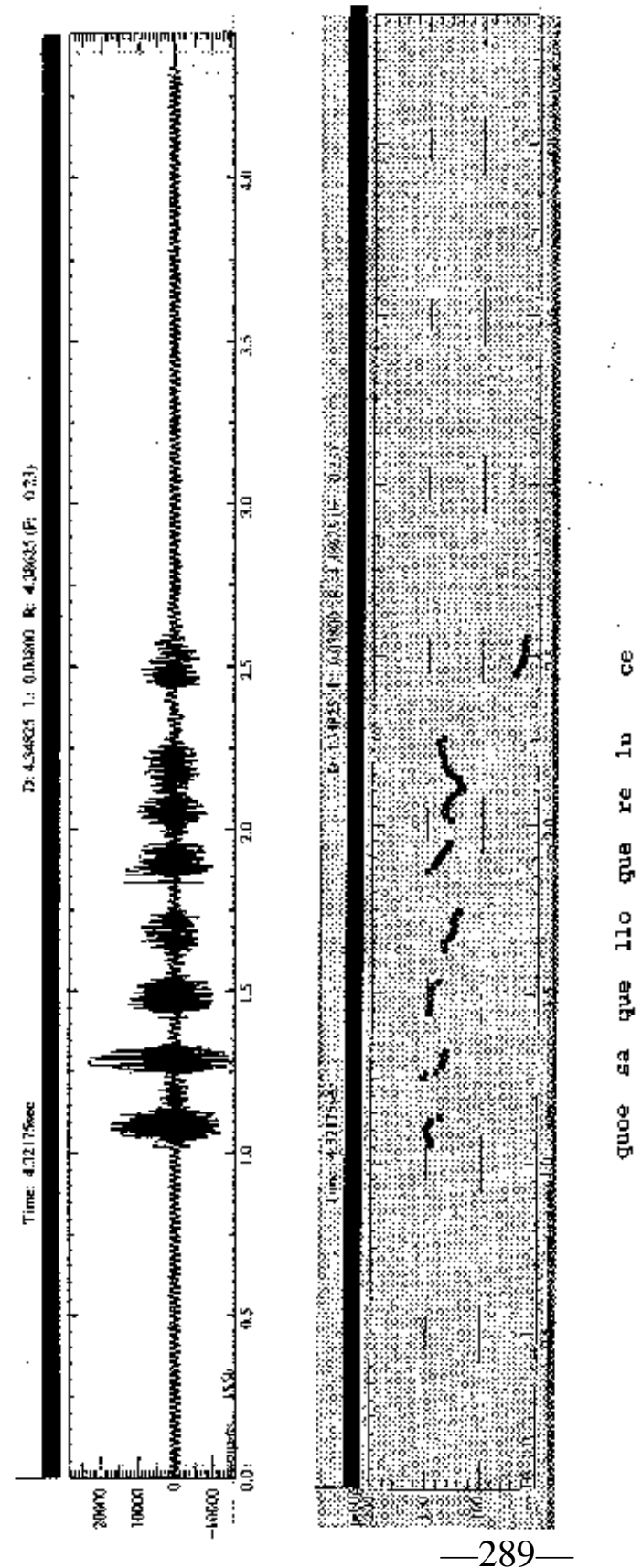
$-290-$ 

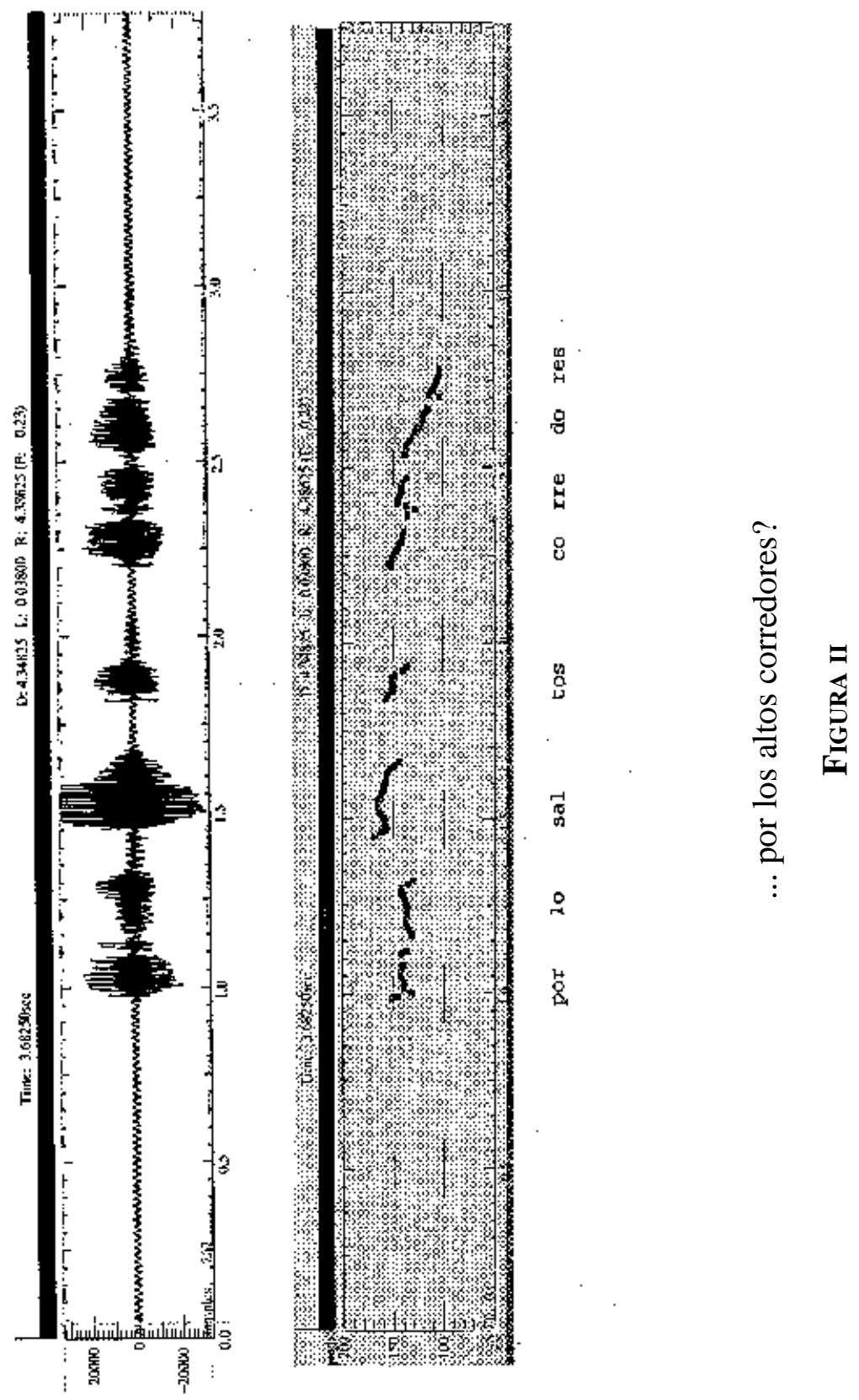

$\stackrel{\leftrightarrow}{\mathrm{g}}$ 
$-292-$ 

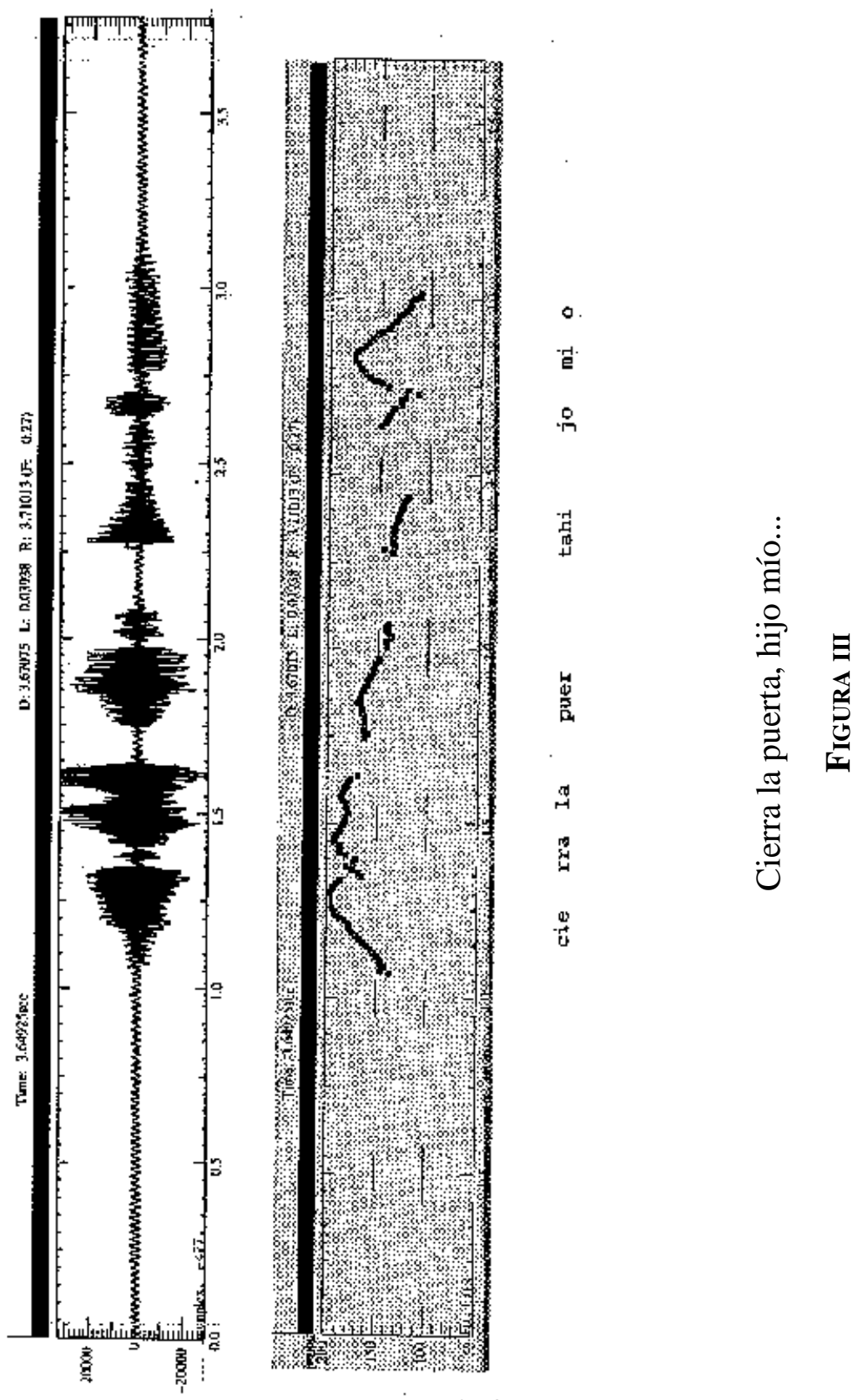

$\stackrel{\substack{0 \\-1}}{u}$ 
$-294-$ 

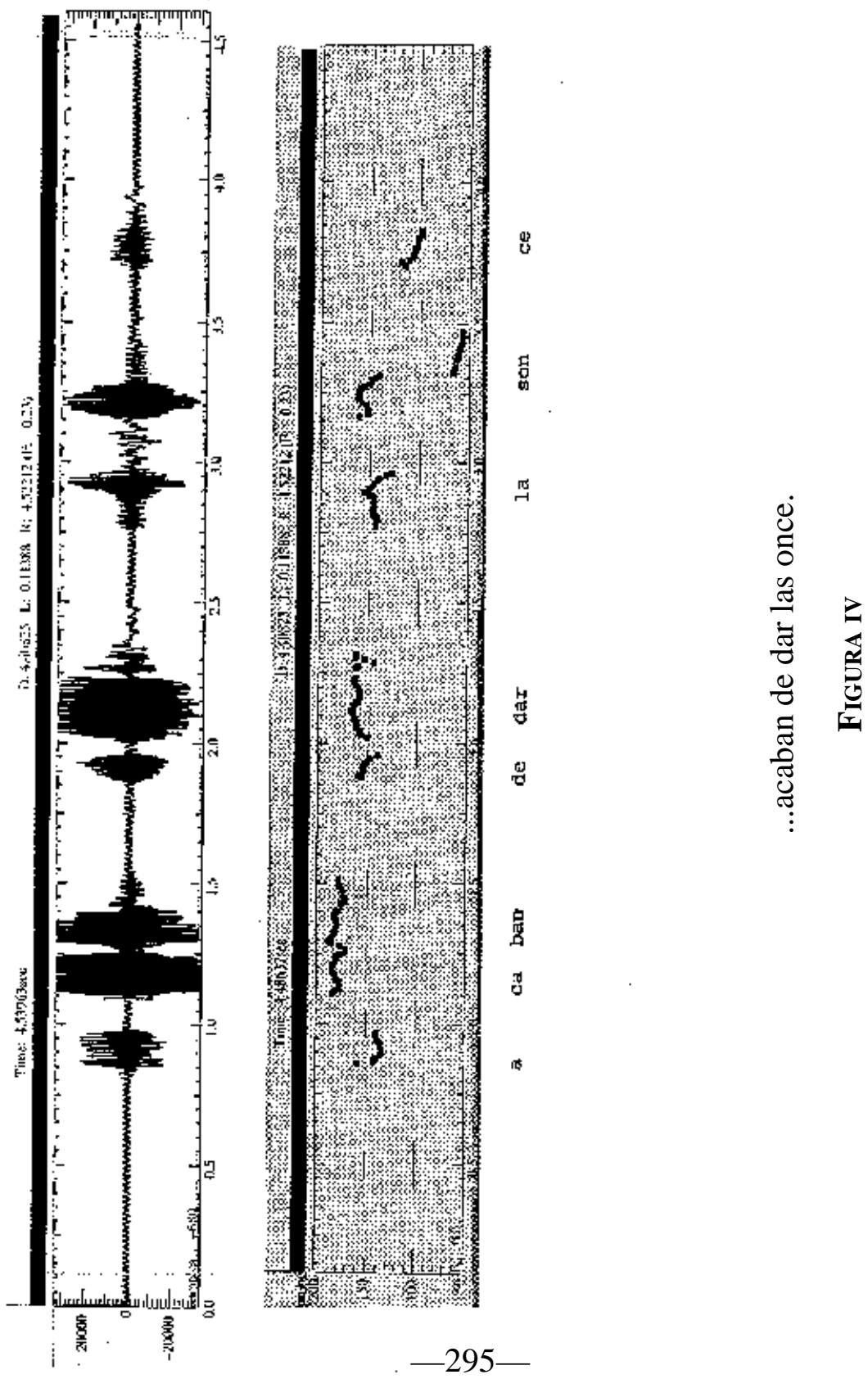
$-296-$ 

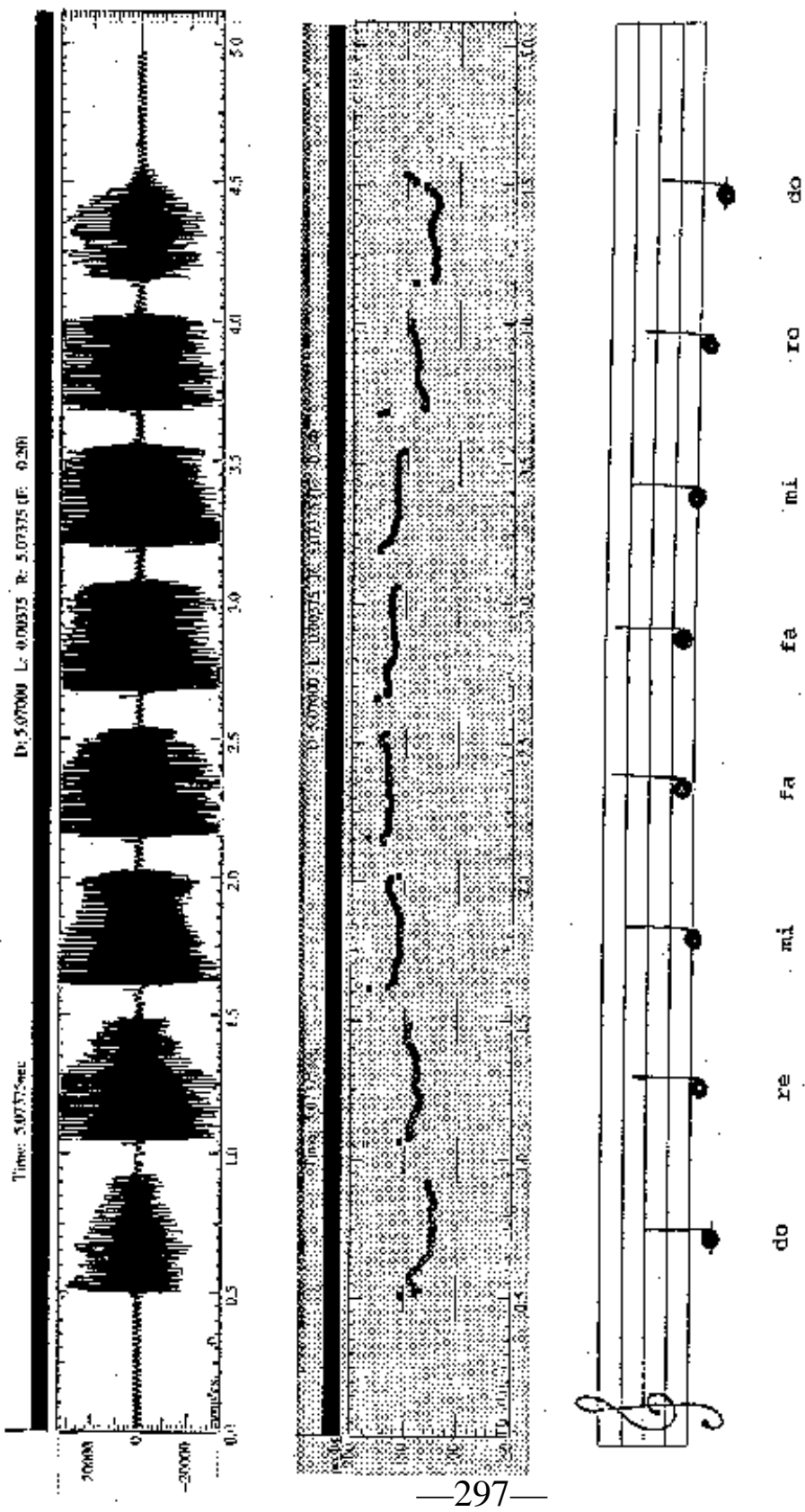
$-298-$ 


\section{REFERENCIAS BIBLIOGRÁFICAS}

ABERCROMBIE, D.: Elements of General Phonetics, Edinburgh University Press, Edimburgo, 1967.

ALLEN, W.S.:Accent and Rhythm, Cambridge University Press, Cambridge, 1973.

ALMEIDA, M.: "Patrones rítmicos del español. Isocronía y alternancia”, Estudios Filológicos, 29, 1994, pp. 118-123.

BAKER, D. (ed.): Meter in English: A Critical Engagement, The University of Arkansas Press, Fayetteville, 1996.

BAUSI, F. y MARTELLI, M.: La metrica italiana, Le Lettere, Florencia, 1993.

BELTRAMI, P. G.: Gli strumenti della poesia. Guida alla metrica italiana, Il Mulino, Bolonia, 1996.

BENOT, E.: Prosodia castellana i versificación, 3 vols., Juan Muñoz Sánchez, Madrid, s.a.[1892]; ed. facsímil con introducción y notas de E. Torre, Rhythmica, Anejo I, Sevilla, 2003.

CANELLADA, M. J. y MADSEN, J. K.: Pronunciación del español. Lengua hablada y literaria, Castalia, Madrid, 1987.

CANTIN I MAS, M. y RÍOS MESTRE, A.: “Análisis experimental del ritmo de la lengua catalana”, Anuario del Seminario de Filología Vasca "Julio de Urquijo", XXV, 2, 1991, pp. 487- 513.

CHATMAN, S.: A Theory of Meter, Mouton, La Haya, 1965.

COUPER-KUHLEN, E.: English Speech Rhythm, John Benjamins, Amsterdam, 1993.

CUENCA VILLARÍN, M. H.: Estudio comparativo del ritmo inglés / español, Tesis Doctoral, Universidad de Sevilla, 1997.

DE ROSA, F. y SANGIRARDI, G.: Introduzione alla metrica italiana, Sansoni, Milán, 1996.

DI GIROLAMO, C.: Teoria e prassi della versificazione, Il Mulino, Bolonia, 1976.

FUBINI, M.: Metrica e poesia. Lezioni sulle forme metriche italiane, Feltrinelli, Milán, 1975.

GILI GAYA, S.: Elementos de fonética general, Gredos, Madrid, 1975.

GRAMMONT, M.: Petit traité de versification française, Armand Colin, París, 1974.

GROSS, H.: The Structure of Verse: Modern Essays on Prosody, Echo Press, Nueva York, 1979.

HALLE, M. y KEYSER, S. J.: English Stress: Its Forms, its Growth, and its Role in Verse, Harper \& Row, Nueva York, 1971. 
HALLE, M. y VERGNAUD, J.-R.: An Essay on Stress, MIT Press, Cambridge, 1987.

HARRIS, J. W.: Syllable Structure and Stress in Spanish: A Nonlinear Analysis, MIT Press, Cambridge, 1983. Trad. española: La estructura silábica y el acento en español: Análisis no lineal, Visor, Madrid, 1991.

HAYES, B.: Metrical Stress Theory. Principles and Case Studies, The University of Chicago Press, Chicago, 1995.

KAGER, R.: A Metrical Theory of Stress and Destressing in English and Dutch, Foris, Dordrecht, 1989.

KIBÉDI VARGA, A.: Les constantes du poème. Analyse du langage poétique, Picard, París, 1977.

LINDBLOM, B.E.F.: "Some Temporal Regularities of Spoken Swedish", en G. Fant \& A.A. Tatham (eds.): Auditory Analysis and Perception of Speech, Academic Press, Londres, Nueva York y San Francisco, 1975, pp. 387-396.

LOTZ, J.: "Metrics", en Th. A. Sebeok (ed.): Current Trends in Linguistics, XII, Mouton, La Haya, 1974, pp. 963-978.

MENICHETTI, A.: "Per un nuovo manuale di metrica italiana”, Metrica, IV, 1986, pp. 7-19.

NAVARRO TOMÁS, T.: Manual de pronunciación española, $6^{\mathrm{a}}$ ed. corregida, Hafner, Nueva York, 1966.

NYÉKI, L.: "Le rythme linguistique en français et en hongrois (Essai de prosodie contrastive)", Langue Française, 19, 1973, pp. 120-142.

ORLANDO, S.: Manuale di metrica italiana, Bompiani, Bolonia, 1993.

PARDO, A.: "El endecasílabo con acentos en $6^{\mathrm{a}}$ y $7^{\mathrm{a}}$ sílabas", en Th. Gomez (ed.): Variations autour de la poésie, Hommage à Bernard Sesé, Vol. 2, C.R.I.I.A., Nanterre, 2001, pp. 87-108.

PAZZAGLIA, M.: Manuale di metrica italiana, Sansoni, Florencia, 1990.

POINTON, G.: A Contribution to the Study of Rhythm in Spanish, University of Edinburgh, Edimburgo, 1978.

QUILIS, A. y FERNÁNDEZ, J.A.: Curso de fonética y fonología españolas, C.S.I.C., Madrid, 1982.

REAL ACADEMIA ESPAÑOLA: Esbozo de una nueva Gramática de la Lengua Española, Espasa-Calpe, Madrid, 1973.

SPONGANO, R.: Nozioni ed esempi di metrica italiana, Pàtron, Bolonia, 1974.

TOLEDO, G. A.: El ritmo en el español. Estudio fonético con base computacional, Gredos, Madrid, 1988.

TOLEDO, G. A.: "Alternancia y ritmo en el español: habla espontánea”, Estudios Filológicos, 31, 1996, pp. 119-127.

TORRE, E.: "El verso y sus elementos”, en E. Torre y M. A. Vázquez: Fundamentos de Poética Española, Alfar, Sevilla, 1986, pp. 23-58.

TORRE, E.: El ritmo del verso (Estudios sobre el cómputo silábico y la distribución acentual, a la luz de la Métrica Comparada, en el verso español moderno), Universidad de Murcia, Murcia, 1999; reimp. 2002.

TORRE, E.: Métrica española comparada, Universidad de Sevilla, Sevilla, 2000; reimp. 2002. 
TRAGER, G. L. y SMITH, H. L.: An Outline of English Structure, University of Oklahoma Press, Oklahoma, 1951.

VISCH, E.: A Metrical Theory of Rhythmic Stress Phenomena, Foris, Dordrecht, 1990. 